\title{
Indications for the Nonexistence of Three-Neutron Resonances near the Physical Region
}

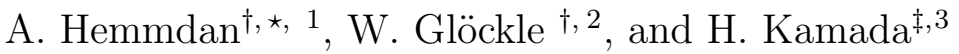 \\ ${ }^{\dagger}$ Institut für Theoretische Physik II, Ruhr-Universität Bochum, D-44870 Bochum, Germany \\ ${ }^{\star}$ Department of Physics, Faculty of Science, South Valley University, Aswan, Egypt \\ ${ }^{\ddagger}$ Department of Physics, Faculty of Engineering, Kyushu Institute of Technology, \\ 1-1 Sensuicho, Tobata, Kitakyushu 804-8550, Japan
}

\begin{abstract}
The pending question of the existence of three-neutron resonances near the physical energy region is reconsidered. Finite rank neutron-neutron forces are used in Faddeev equations, which are analytically continued into the unphysical energy sheet below the positive real energy axis. The trajectories of the three-neutron $S$-matrix poles in the states of total angular momenta and parity $J^{\pi}=\frac{1}{2}^{ \pm}$and $J^{\pi}=\frac{3}{2}^{ \pm}$are traced out as a function of artificial enhancement factors of the neutron-neutron forces. The final positions of the $S$-matrix poles removing the artificial factors are found in all cases to be far away from the positive real energy axis, which provides a strong indication for the nonexistence of nearby three-neutron resonances. The pole trajectories close to the threshold $E=0$ are also predicted out of auxiliary generated three-neutron bound state energies using the Padé method and agree very well with the directly calculated ones.
\end{abstract}

PACS numbers: 21.45.+v,24.30.Gd,25.70.Ef

\footnotetext{
1 email: Amel.Hemdan@tp2.ruhr-uni-bochum.de

${ }^{2}$ email: Walter.Gloeckle@tp2.ruhr-uni-bochum.de

3 email: kamada@mns.kyutech.ac.jp
} 


\section{Introduction}

Controversial experimental hints on the existence of three-neutron (3n) resonances [1-4] and more recently even on four-neutron resonances [5] occurred again and again. No definite experimental conclusion on $3 n$ resonances seem to exist. On the theoretical side we are aware of various attempts to investigate states of $3 n$ 's but also here the situation is pretty unsettled. Obviously the optimal theoretical present day insight would be if the most modern neutronneutron $(n n)$ forces from the set of high precision $N N$ potentials together with $3 n$ forces of the $2 \pi$-exchange type (Tucson-Melbourne [6] or Urbana type [7]) would be used and the energy eigenvalues of the $3 n$ Schrödinger equation according to resonance boundary conditions would be determined in the unphysical energy sheet adjacent to the positive real energy axis. Unfortunately we can not provide such an insight right now but think we can improve at least on the existing studies we are aware of.

One of the possibly first studies on that list is a solution of the $3 n$ Faddeev equation based on a Yamaguchi rank $1 \mathrm{nn}$ force in the state ${ }^{1} S_{0}$ [8]. Only the $3 n$ states of total angular momenta $J^{\pi}=1 / 2^{-}$and $3 / 2^{-}$(degenerate in this case) have been studied. The $n n$ force was artificially enhanced such that two and three neutrons were bound. Then the enhancement was reduced which has the consequence that both bound state energies move towards $E=0$. For the forces used it happened that the $3 n$ binding energy moves faster than the $2 n$ binding energy and thus hits the dineutron-neutron threshold to the left of $E=0$. The trajectory of the $3 n$ bound state energy continues then into the unphysical sheet below the dineutron-neutron cut. In this manner a $3 n$ resonance occurs, below the $3 n$ break-up threshold at $E=0$. Further decreasing the enhancement factor the $3 n$ resonance trajectory moves up again exactly towards $E=0$ where it meets with the $2 n$ bound state energy. Both energies coincide exactly at $E=0$ corresponding still to an enhancement factor larger than 1. In further decreasing the potential strength till one reaches the physical value 1 the vanishing $2 n$ bound state energy turns into the well established $2 n$ virtual state energy in the second sheet on the negative real energy axis and the $3 n$ resonance disappears in a unphysical sheet which is different from the sheet adjacent to the positive real axis. In other words the complex resonance energy of that $3 n$ resonance for $J^{\pi}=1 / 2^{-}$and $3 / 2^{-}$has no positive real part and therefore can not be felt for positive three-neutron energies. We have to emphasise that this refers to a $n n$ force acting only in the state ${ }^{1} S_{0}$ and it will change if additional $n n$ force components will be added as will be shown in this paper.

A very much related study has been performed in [9] with essentially the same result as in [8]. Using just bound state techniques [10] the necessary enhancement factors on the $n n$ forces in $S$ - and $P$-waves have been determined to bind 3 neutrons near zero energy. Based on the Reid potential [11] enhancement factors of the order of 4 have been found which make low lying $3 n$ resonances quite unlikely.

Another theoretical investigation [12] we are aware of for the state $J^{\pi}=1 / 2^{-}$has been performed on the basis of a hyperspherical harmonic expansion. Local forces like the ones of Mafliet Tjon have been used and this only in the $n n$ state ${ }^{1} S_{0}$. The expansion was truncated and the zeros of the Jost function were determined. It resulted a resonance energy around $E=-4.9-i 6.9 \mathrm{MeV}$ in an unphysical sheet. The authors found a strong sensitivity to the choice of the potential.

A further investigation [13] relied on a variational ansatz and used the complex scaling method 
which allows to treat a resonance problem like a bound state one. The Minnesota effective $n n$ force [14] together with tensor forces [15] have been applied. The authors find as the only candidate for a $3 n$ resonance the state $J^{\pi}=3 / 2^{+}$and a prediction for its energy of $E=14-i 13$ $\mathrm{MeV}$.

Finally in a more recent work [16] the Faddeev equation in configuration space has been solved for the states $J^{\pi}=1 / 2^{-}$and $3 / 2^{ \pm}$using the more realistic forces by Gogny et al. [17] and the Reid93 [18] potential in the $2 n$ states ${ }^{1} S_{0},{ }^{3} P_{0}$ and ${ }^{3} P_{2}-{ }^{3} F_{2}$. Choosing proper boundary conditions according to $3 n$ resonances the complex energy eigenvalues are determined starting again from artificially enhanced forces and reducing their strengths gradually. Unfortunately with increasing negative imaginary parts of the complex resonance energies numerical instabilities occurred and the trajectories could not be followed up until the physical values for the enhancement factors have been reached.

Now we would like to improve on all that by solving the Faddeev equations with low rank $n n$ forces in all relevant partial wave states exactly and determining the final resonance positions for the actual force strength as prescribed by $n n$ phase shifts (assumed to be identical to the strong $p p$ phase shifts). In the light of our results we shall also comment on the previous findings.

This paper is organised as follows. In section II we briefly review the set of coupled Faddeev eigenvalue equations for a $3 n$ system based on finite rank forces and mention the steps needed for an analytical continuation into the unphysical energy sheet adjacent to the positive real axis. The dynamical $n n$ force input and the resulting $3 n$ resonance trajectories for the states $J^{\pi}=1 / 2^{ \pm}$and $3 / 2^{ \pm}$are presented in section III. In section IV we show how the part of the $3 n$ resonance trajectory close to the threshold $E=0$ can be predicted with the help of Padé approximant's from a set of $3 n$ bound state energies. This serves as a test for our results obtained through the analytical continuation of the Faddeev equation. Though the final $3 n$ resonance positions could not be reached with that method, we think this illustration should be of interest since it works beautifully for resonances close to the first threshold and requires only bound state techniques. We summarise in section $\mathrm{V}$.

\section{Formalism}

The Faddeev equation for three identical nucleons has the well known form [19]

$$
\psi=G_{0} t P \psi,
$$

where $G_{0}$ is the free $3 N$ propagator, $t$ the $N N$-matrix and $P$ the sum of a cyclical and anticyclical permutation of 3 objects. We regard that equation in a momentum space representation and partial wave decomposed. It results a set of coupled equations in two variables, $\mathrm{p}$ and $\mathrm{q}$, which are the magnitudes of two relative momenta. In the present investigation we shall use $t$ matrices of finite rank. The set of two-dimensional equations changes then into a set of coupled one-dimensional ones. They have the well known form

$$
\begin{aligned}
\theta_{\alpha}^{j}(q)= & \int_{0}^{\infty} d q^{\prime} q^{\prime}{ }^{2} \sum_{\alpha \prime} \sum_{k m} \int_{-1}^{1} d x \frac{g_{j}^{\tilde{\alpha}}\left(\pi_{1}\right)}{\pi_{1}^{l}} G_{\alpha \alpha \prime}\left(q, q^{\prime}, x\right) \\
& \frac{1}{\left(E-\frac{q^{2}}{M}-\frac{q^{2}}{M}-\frac{q q \prime x}{M}\right)} \frac{g_{k}^{\tilde{\alpha}^{\prime}}\left(\pi_{2}\right)}{\pi_{2}^{l \prime}} \tau_{k m}^{\tilde{\alpha}^{\prime}}(z \prime) \theta_{\alpha \prime}^{m}\left(q^{\prime}\right)
\end{aligned}
$$


and $\theta$ is connected to $\psi$ via

$$
\psi_{\alpha}(p, q)=\frac{1}{E-\frac{p^{2}}{M}-\frac{3 q^{2}}{4 M}} \sum_{j, k} g_{j}^{\tilde{\alpha}}(p) \tau_{j, k}^{\tilde{\alpha}}(z) \theta_{\alpha}^{k}(q) .
$$

The underlying two-body $t$-matrices are

$$
t(p, p \prime, z)=\sum_{i, j} g_{i}(p) \tau_{i, j}(z) g_{j}(p \prime)
$$

The corresponding form factors $g(p)$ depend on the obvious two-body quantum numbers $\tilde{\alpha} \equiv$ $(l s j)$. The two-body subsystem energy $z=E-\frac{3 q^{2}}{4 M}$ is given in terms of the total energy $E$ and the kinetic energy of relative motion of the third particle. Further $\alpha$ stands for the string of three-body quantum numbers

$$
\alpha \equiv(l s) j\left(\lambda \frac{1}{2}\right) I(j I) J\left(t \frac{1}{2}\right) T
$$

For all those by now standard notations we refer to [19]. Finally $G_{\alpha \alpha \prime}\left(q, q^{\prime}, x\right)$ are geometrical coefficients arising from the partial wave representation of the permutation operator $P$ and $\pi_{1}$ and $\pi_{2}$ are shifted arguments related to $P$ and depend on $q, q$ and $x$, see [19] for details.

We are interested in $3 n$ bound state and resonance energies, the latter ones emerging from bound state energies when the artificially increased interaction strength is reduced. Since two neutrons are not bound in nature the only unphysical sheet of interest is the one which can be reached through the cut along $0 \leq E<\infty$. It will be denoted in the following simply as the unphysical sheet. While for $E<0$ there is no singular denominator, for $E>0$ the free propagator in Eq. (2) is not defined. The well established manner to analytically continue the coupled set of equations in the energy into the unphysical sheet is to deform the path of integration into the lower half plane. This has been described for instance in [8] and [20]. The singularities which are avoided in this manner arise from the free propagator and the form factors. The $\tau$-function is not singular for energies $z=E-\frac{3 q^{2}}{4 m}$ in the unphysical sheet under investigation. A typical path in the complex $q$-plane (for both $q$ and $q$ ) together with domains of singularity arising from the free propagator for the example $E=10.0-i 10.0 \mathrm{MeV}$ is shown in Fig. 1.

The strategy now as pointed out in the introduction is to first enhance the $n n$ forces such that a $3 n$ bound state exists. At the same time also two-neutron bound states might exist. Then by suitably chosen enhancement factors one can achieve that the two-body bound state energies coincide and move together towards $E=0$, when the enhancements are reduced. In all cases we studied the $3 n$ bound state energies stayed always to the left of the two-body bound state energies until they reached $E=0$. This was not the case in [8], where for the degenerate states $1 / 2^{-}$and $3 / 2^{-}$only the ${ }^{1} S_{0}$ force had been kept. Here we include on top $P$ - and $D$ wave forces and such a coincidence of $3 n$ and $2 n$ bound state energies does not occur. Once the two-neutron bound state energies have disappeared we still have a $3 n$ bound state. Then reducing the enhancement factors further the $3 n$ bound state energy will approach $E=0$ and then the energy eigenvalue $E$ will dive into the lower half plane of the unphysical sheet. There we shall follow its trajectory until the physical strengths of the $n n$ forces are reached. This defines the final positions of the $3 n$ resonances. It is well known and easily seen that what we call $3 n$ resonance energies are poles of the $3 n S$-matrix. 


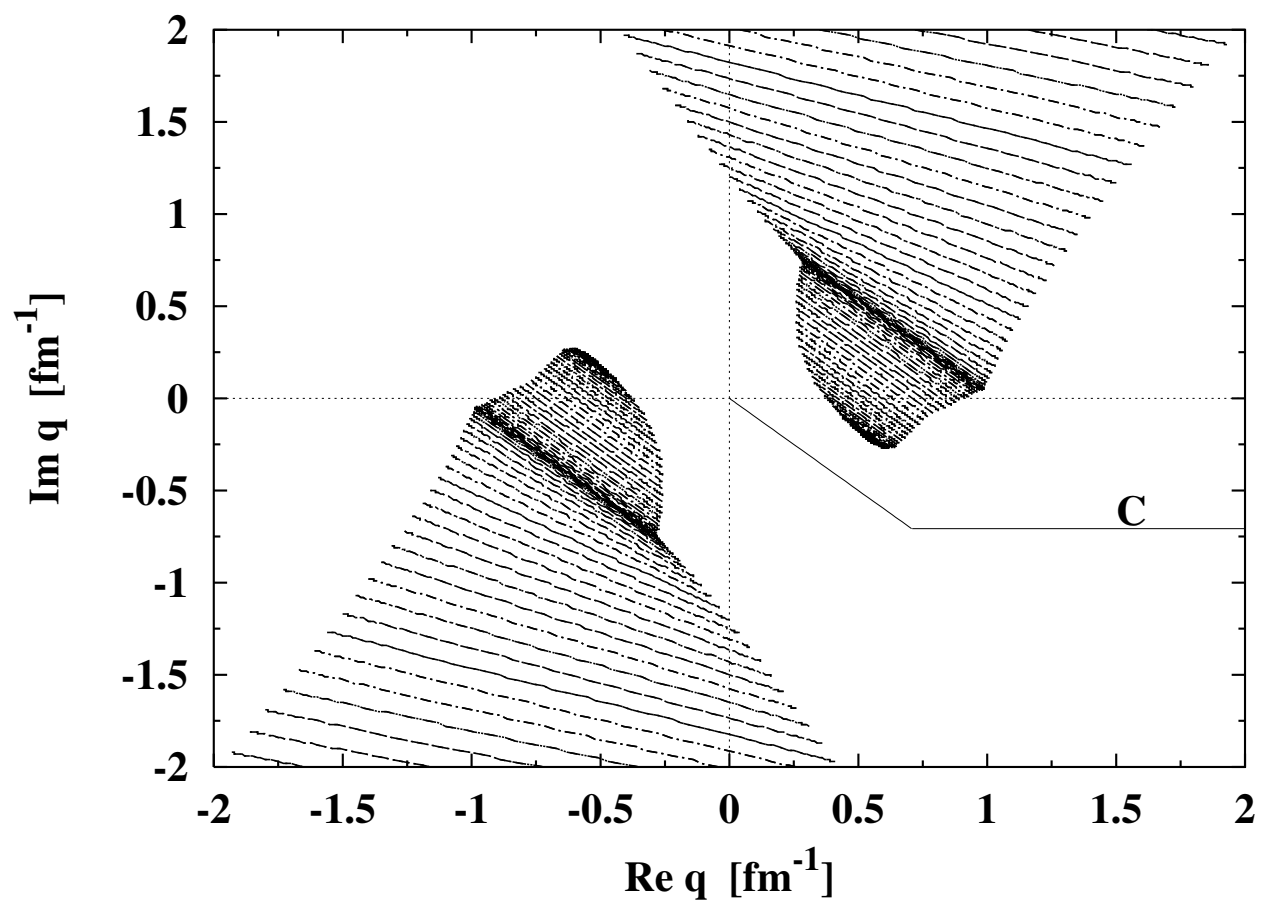

Figure 1: The regions of singularities from the free propagator for the complex energy $E=$ $10.0-i 10 \mathrm{MeV}$ and a path of integration $\mathrm{C}$.

\section{Results}

We use $n n$ forces of rank 2. They are given in Appendix A. They describe $p p$ phase shifts (without Coulomb force) reasonably well as documented in the Appendix. We restrict our study to the states ${ }^{1} S_{0},{ }^{3} P_{0},{ }^{3} P_{2}$ and ${ }^{1} D_{2}$. The coupling between ${ }^{3} P_{2}$ and ${ }^{3} F_{2}$ is neglected. The $n n$ force in the state ${ }^{3} P_{1}$ is repulsive and therefore we did not include it in the main investigation, since it has to be expected, that it will move the $3 n$ resonance position further away from the positive real energy axis. At the end we shall consider its effect and show that its influence has indeed repulsive character but only a marginal one and could therefore indeed be neglected.

We shall investigate now the 4 possible states $J^{\pi}=1 / 2^{ \pm}$and $J^{\pi}=3 / 2^{ \pm}$in turn.

\subsection{The state $3 / 2^{-}$}

We use up to 10 three-neutron channels which are displayed in Table 1 . To reach a $3 n$ bound state it is sufficient to enhance just the ${ }^{3} P_{2} n n$ force and to keep all the other force components at their physical values. As an example for an enhancement factor we quote $\lambda_{3} P_{2}=3.5$ where the 3 neutrons in a 10 channel calculation are bound at $-7.79 \mathrm{MeV}$. The threshold energy $E=0$ is reached for $\lambda_{{ }^{3} P_{2}}=3.23$. For the sake of future comparisons we provide a few intermediate $3 n$ resonance positions in Table 2 . The $3 n$ resonance trajectory is shown in Fig. 2 for a 10 channel calculation. The trajectories for a smaller number of channels are very similar. The final resonance positions for an increasing number of channels in the order given in Table 1 are displayed in Table 3. Adding more $n n$ force components will certainly not change the final 
Table 1: The partial wave quantum number for the three neutron state $J^{\pi}=\frac{3}{2}^{-}$.

\begin{tabular}{||c|c|c|c|c|c||}
\hline \hline$n n$ state & $l$ & $s$ & $j$ & $\lambda$ & $I$ \\
\hline \hline${ }^{1} S_{0}$ & 0 & 0 & 0 & 1 & $3 / 2$ \\
${ }^{3} P_{2}$ & 1 & 1 & 2 & 0 & $1 / 2$ \\
${ }^{3} P_{0}$ & 1 & 1 & 0 & 2 & $3 / 2$ \\
${ }^{3} P_{2}$ & 1 & 1 & 2 & 2 & $3 / 2$ \\
${ }^{3} P_{2}$ & 1 & 1 & 2 & 2 & $5 / 2$ \\
${ }^{1} D_{2}$ & 2 & 0 & 2 & 1 & $1 / 2$ \\
${ }^{1} D_{2}$ & 2 & 0 & 2 & 1 & $3 / 2$ \\
${ }^{1} D_{2}$ & 2 & 0 & 2 & 3 & $5 / 2$ \\
${ }^{3} P_{2}$ & 1 & 1 & 2 & 4 & $7 / 2$ \\
${ }^{1} D_{2}$ & 2 & 0 & 2 & 3 & $7 / 2$ \\
\hline \hline
\end{tabular}

position in a significant manner in the sense that the position would come somewhere close to the positive real energy axis. If one adds the ${ }^{3} P_{1} n n$ force, which is of repulsive nature, we have 13 channels and the final resonance position shifts by $-1.79-i 2.87 \mathrm{MeV}$, which is marginal. In Ref.[16] which can be considered to be the most realistic approach towards three-neutron resonances carried out so far the rather realistic $n n$ forces by Gogny [17] and Reid93 [18] have been used. In contrast to our simplified forces they also include the tensor force induced coupling ${ }^{3} P_{2}-{ }^{3} F_{2}$. For the case of the Gogny potential the enhancement factor for the ${ }^{3} P_{2}-{ }^{3} F_{2} n n$ force component was about 4.4 to have $3 n$ 's bound at zero energy, while for our simplified force this was the smaller value of 3.5. In [16] reducing $\lambda^{3} P_{2}-{ }^{3} F_{2}$ to 3.7 yields a resonance energy of $4.95-i 3.75 \mathrm{MeV}$, while at this value our $3 n$ system is still bound. Thus one might conjecture that in the more realistic case the $3 n$ resonance would be even further shifted away from the positive real energy axis. On the other hand the motion of our resonance energy for a change of $\Delta \lambda=4.4-3.7=0.7$ from the threshold $E=0$ (corresponding to an enhancement factor $2.8)$ is $5.58-i 4.04 \mathrm{MeV}$, which is slightly larger. Therefore a direct quantitative comparison is not possible, though qualitatively the results are similar. This is also true for the case of Reid93, also considered in [16], where the enhancement factor $\lambda_{3} P_{2}-{ }^{3} F_{2}=3.25$ yields a resonance position of $5.30-i 3.53 \mathrm{MeV}$.

\subsection{The state $3 / 2^{+}$}

Again we use up to 10 channels, which are displayed in Table 4. In order to have a $3 n$ bound state we had to enhance the ${ }^{3} P_{0}$ and ${ }^{3} P_{2} n n$ forces while the ${ }^{1} S_{0}$ and ${ }^{1} D_{2}$ forces could be kept at their physical values. For the enhancement factors $\lambda_{3} P_{2}=3.5$ and $\lambda^{3} P_{0}=5.02 n$ bound 
Table 2: Intermediate $3 n$ resonance positions together with the enhancement factor $\lambda_{3} P_{2}$ for a 10 channel calculation and $J^{\pi}=\frac{3}{2}^{-}$.

\begin{tabular}{||c|c|}
\hline \hline$\lambda{ }_{P_{2}}$ & $E_{3 n}^{\text {res. }}[\mathrm{MeV}]$ \\
\hline \hline 3.22 & $0.20-i 0.003$ \\
3.20 & $0.57-i 0.03$ \\
3.00 & $3.51-i 1.45$ \\
2.00 & $5.40-i 19.17$ \\
1.50 & $-1.95-i 28.87$ \\
1.00 & $-12.13-i 37.96$ \\
\hline \hline
\end{tabular}

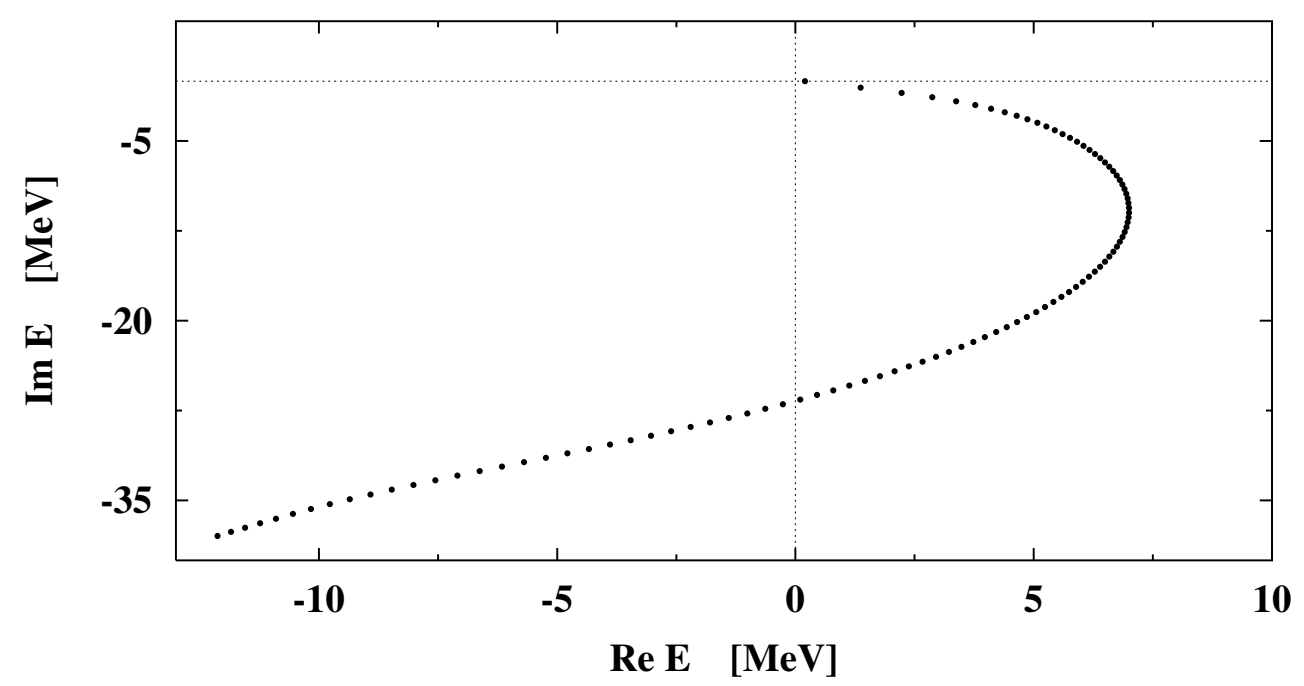

Figure 2: The resonance pole trajectory for the state $3 / 2^{-}$.

Table 3: The final $3 n$ resonance positions for the state $J^{\pi}=\frac{3}{2}^{-}$including different number of channels.

\begin{tabular}{||c|c||}
\hline \hline Number of channels & Final resonance positions \\
\hline \hline 2 & $-12.81-i 33.22$ \\
3 & $-13.14-i 33.21$ \\
4 & $-13.11-i 33.17$ \\
5 & $-13.33-i 32.91$ \\
6 & $-14.28-i 33.37$ \\
7 & $-12.43-i 37.65$ \\
8 & $-12.31-i 37.74$ \\
9 & $-12.31-i 37.74$ \\
10 & $-12.13-i 37.96$ \\
\hline \hline
\end{tabular}


Table 4: The partial wave quantum number for the three neutron state $J^{\pi}=\frac{3}{2}^{+}$.

\begin{tabular}{||c|c|c|c|c|c||}
\hline \hline$n n$ state & $l$ & $s$ & $j$ & $\lambda$ & $I$ \\
\hline \hline${ }^{1} S_{0}$ & 0 & 0 & 0 & 2 & $3 / 2$ \\
${ }^{3} P_{0}$ & 1 & 1 & 0 & 1 & $3 / 2$ \\
${ }^{3} P_{2}$ & 1 & 1 & 2 & 1 & $1 / 2$ \\
${ }^{3} P_{2}$ & 1 & 1 & 2 & 1 & $3 / 2$ \\
${ }^{1} D_{2}$ & 2 & 0 & 2 & 0 & $1 / 2$ \\
${ }^{3} P_{2}$ & 1 & 1 & 2 & 3 & $5 / 2$ \\
${ }^{3} P_{2}$ & 1 & 1 & 2 & 3 & $7 / 2$ \\
${ }^{1} D_{2}$ & 2 & 0 & 2 & 2 & $3 / 2$ \\
${ }^{1} D_{2}$ & 2 & 0 & 2 & 2 & $5 / 2$ \\
${ }^{1} D_{2}$ & 2 & 0 & 2 & 4 & $7 / 2$ \\
\hline \hline
\end{tabular}

states for ${ }^{3} P_{2}$ and ${ }^{3} P_{0}$ just disappear and the 3 neutrons are bound at $-6.55 \mathrm{MeV}$. Then we reduce both enhancement factors. The way this is done is in principle arbitrary. Again for future work we provide some intermediate $3 n$ resonance positions along the trajectory in the unphysical sheet in Table 5. The corresponding trajectory is shown in Fig. 3. The dependence of the final position on the number of channels is displayed in Table 6 . Like for $3 / 2^{-}$the final $S$-matrix pole position is far away from the positive real energy axis. Again the effect of ${ }^{3} P_{1}$ $n n$ force is marginal (a shift of $-0.80-i 0.42 \mathrm{MeV}$ ).

Now we compare to the work in Ref. [16]. The enhancement factors for the Gogny potential for the ${ }^{3} P_{0}$ and ${ }^{3} P_{2}-{ }^{3} F_{2} n n$ force components are about 7.7 and 4.9 to generate $n n$ bound states at zero energy. This is larger in both cases than for our simplified potential. Correspondingly according to Ref. [16] for $\lambda^{3} P_{0}=5.0$ and $\lambda^{3} P_{2}-{ }^{3} F_{2}=4.30$ one ends up with a resonance at $5.74-i 1.53 \mathrm{MeV}$, while for such enhancement factors our $n n$ forces still bind the $3 n$ system. Thus also here one is tempted to conjecture that the more realistic Gogny potential would lead to a final resonance position, which is farther away from the real axis than what we find. For the Reid93 potential the corresponding values are $5.93-i 1.55 \mathrm{MeV}$ for $\lambda_{3} P_{0}=4.6$ and $\lambda_{{ }^{3} P_{2}-{ }^{3} F_{2}}=3.50$.

\subsection{The state $1 / 2^{-}$}

The partial wave quantum numbers for that state are shown in Table 7 . In this case there are only 5 channels. It was necessary to enhance the ${ }^{3} P_{2}$ and ${ }^{1} D_{2} n n$ forces to reach a $3 n$ bound state. For $\lambda_{3} P_{2}=3.5$ and $\lambda_{1} D_{2}=6.90$ the $2 n$ bound states just disappear and the $3 n$ system is bound with $-1.90 \mathrm{MeV}$. Again we display some intermediate resonance positions in Table 8 . The final $S$-matrix pole position is significantly further away from the positive real energy axis than for the states $3 / 2^{ \pm}$. The resonance trajectory is shown in Fig. 4 . If one include ${ }^{3} P_{1}$, one has 7 channels and the resonance position shifts by $-4.65-i 0.53 \mathrm{MeV}$ which is insignificant in relation to the value without ${ }^{3} P_{1}$.

This case poses even more difficulties to compare to Ref. [16], since there ${ }^{3} P_{0}$ and ${ }^{3} P_{2}-{ }^{3} F_{2}$ have 
Table 5: Intermediate $3 n$ resonance positions together with the enhancement factor $\lambda_{3_{P_{2}}}$ and $\lambda_{3_{P_{0}}}$ for a 10 channel calculation and $J^{\pi}=\frac{3}{2}^{+}$.

\begin{tabular}{||c|c|c||}
\hline \hline$\lambda{ } P_{2}$ & $\lambda{ } P_{0}$ & $E_{3 n}^{\text {res. }}[\mathrm{MeV}]$ \\
\hline \hline 3.20 & 4.70 & $0.60-i 0.004$ \\
3.00 & 4.50 & $3.21-i 0.96$ \\
2.00 & 3.50 & $6.41-i 14.75$ \\
1.80 & 3.30 & $5.12-i 18.18$ \\
1.00 & 2.50 & $-3.53-i 29.96$ \\
1.00 & 1.00 & $-10.30-i 33.20$ \\
\hline \hline
\end{tabular}

Table 6: The final $3 n$ resonance positions for the state $J^{\pi}=\frac{3}{2}^{+}$including different number of channels.

\begin{tabular}{||c|c||}
\hline \hline Number of channels & Final resonance positions \\
\hline \hline 3 & $-9.82-i 58.37$ \\
4 & $-10.06-i 58.31$ \\
5 & $-10.24-i 33.16$ \\
6 & $-10.25-i 33.17$ \\
7 & $-10.61-i 33.37$ \\
8 & $-10.46-i 33.29$ \\
9 & $-10.35-i 33.23$ \\
10 & $-10.30-i 33.20$ \\
\hline \hline
\end{tabular}

been enhanced while here we found it more natural to enhance ${ }^{3} P_{2}$ and ${ }^{1} D_{2}$ (the latter force component was not included in Ref. [16]). For the enhancements $\lambda^{3} P_{0}=5.5$ and $\lambda_{3} P_{2}-{ }^{3} F_{2}=2.75$ in [16] the $3 n$ resonance turned out to be $2.82-i 2.82 \mathrm{MeV}$. Apparently these factors are still quite large that also here one cannot expect a nearby resonance.

\subsection{The state $1 / 2^{+}$}

Here we used 6 channels displayed in Table 9. Again we need to enhance both the ${ }^{3} P_{2}$ and ${ }^{1} D_{2} n n$ forces by 3.5 and 6.90 , respectively, to get a $3 n$ bound state at $-7.38 \mathrm{MeV}$. Some intermediate resonance positions are shown in Table 10. The corresponding trajectory is shown in Fig. 5. The inclusion of ${ }^{3} P_{1}$ leads to 8 channels and makes a tiny shift to the final resonance position of $-0.36-i 0.02 \mathrm{MeV}$. In Ref. [16] this state has not been considered. 


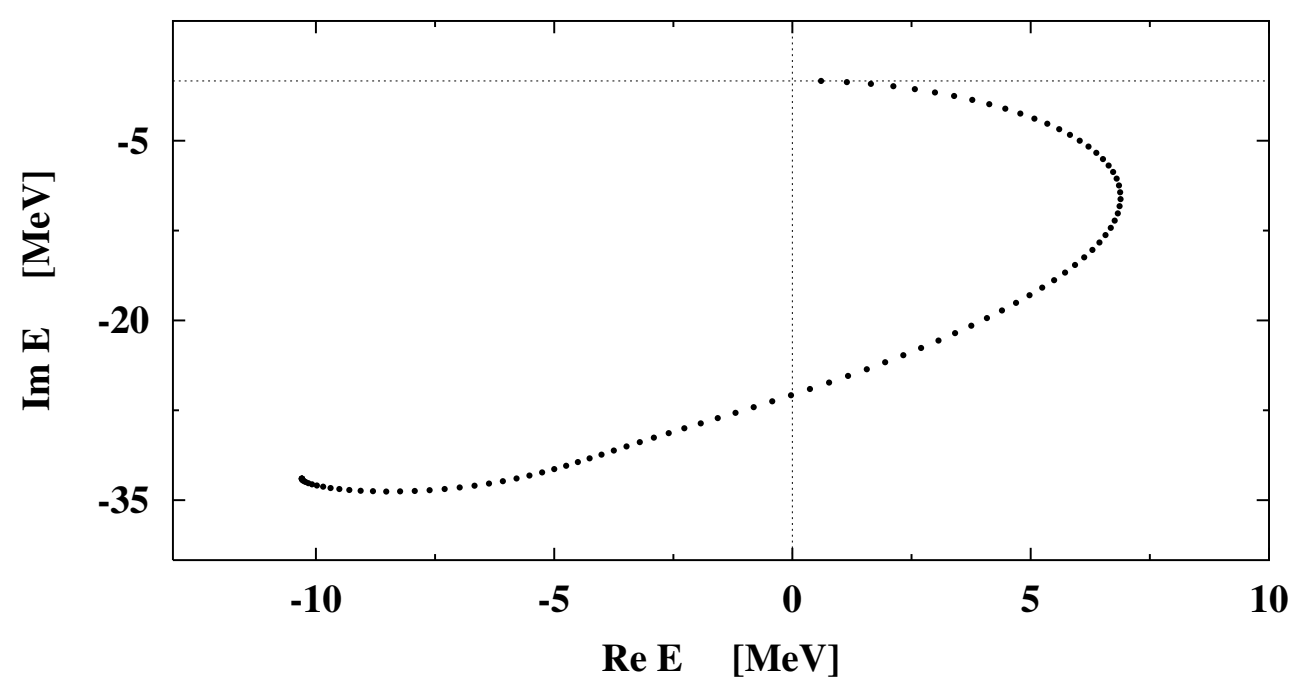

Figure 3: The resonance pole trajectory for the state $3 / 2^{+}$.

Table 7: The partial wave quantum number for the three neutron state $J^{\pi}=\frac{1}{2}^{-}$.

\begin{tabular}{||c|c|c|c|c|c||}
\hline \hline$n n$ state & $l$ & $s$ & $j$ & $\lambda$ & $I$ \\
\hline \hline${ }^{1} S_{0}$ & 0 & 0 & 0 & 1 & $1 / 2$ \\
${ }^{3} P_{0}$ & 1 & 1 & 0 & 0 & $1 / 2$ \\
${ }^{3} P_{2}$ & 1 & 1 & 2 & 2 & $3 / 2$ \\
${ }^{3} P_{2}$ & 1 & 1 & 2 & 2 & $5 / 2$ \\
${ }^{1} D_{2}$ & 2 & 0 & 2 & 1 & $3 / 2$ \\
\hline \hline
\end{tabular}

Table 8: Intermediate $3 n$ resonance positions together with the enhancement factor $\lambda_{3_{P_{2}}}$ and $\lambda_{1_{D_{2}}}$ for a 5 channel calculation and $J^{\pi}=\frac{1}{2}^{-}$.

\begin{tabular}{||c|c|c||}
\hline \hline$\lambda^{{ } P_{2}}$ & $\lambda{ } D_{2}$ & $E_{3 n}^{\text {res. }}[\mathrm{MeV}]$ \\
\hline \hline 3.38 & 6.88 & $0.09-i$ i 0.0002 \\
3.00 & 6.50 & $7.32-i 3.49$ \\
2.00 & 5.50 & $16.44-i 23.16$ \\
1.50 & 5.00 & $14.51-i 32.15$ \\
1.00 & 4.00 & $6.10-i 42.27$ \\
1.00 & 3.00 & $-2.25-i 46.45$ \\
1.00 & 2.00 & $-11.36-i 47.65$ \\
1.00 & 1.00 & $-20.38-i 45.27$ \\
\hline \hline
\end{tabular}




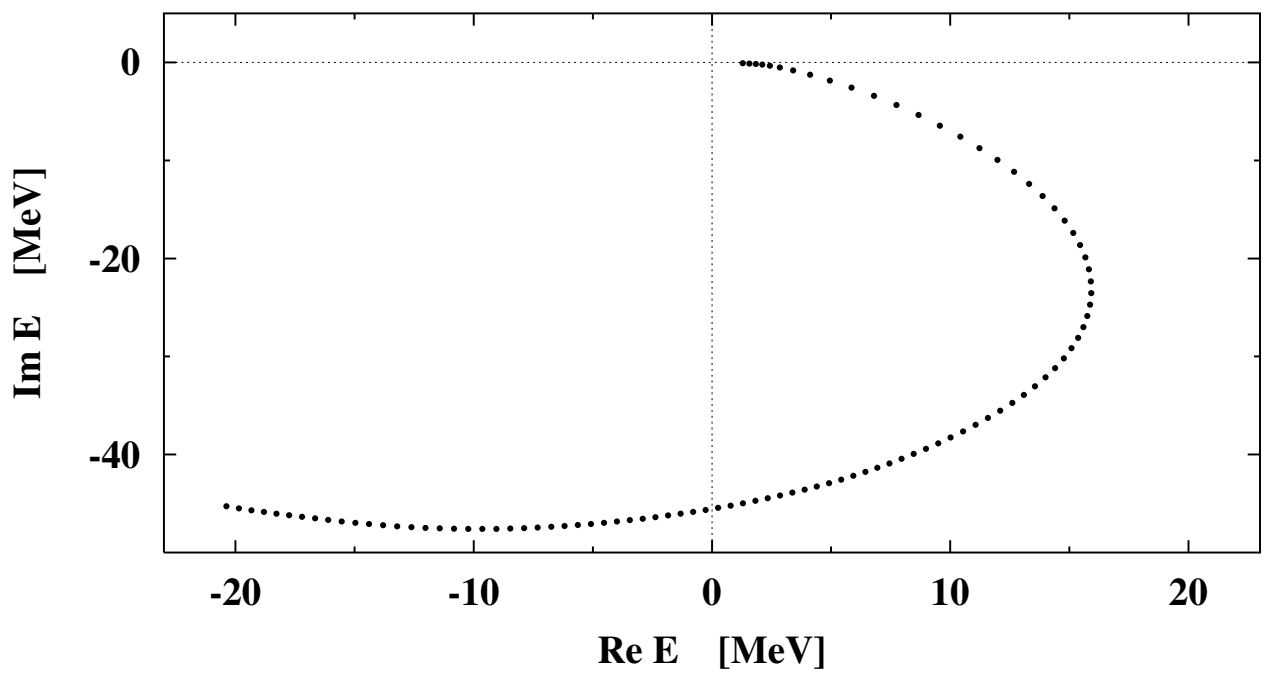

Figure 4: The resonance pole trajectory for the state $1 / 2^{-}$.

Table 9: The partial wave quantum number for the three neutron state $\mathrm{J}^{\pi}=\frac{1}{2}^{+}$.

\begin{tabular}{||c|c|c|c|c|c||}
\hline \hline$n n$ state & $l$ & $s$ & $j$ & $\lambda$ & $I$ \\
\hline \hline${ }^{1} S_{0}$ & 0 & 0 & 0 & 0 & $1 / 2$ \\
${ }^{3} P_{0}$ & 1 & 1 & 0 & 1 & $1 / 2$ \\
${ }^{3} P_{2}$ & 1 & 1 & 2 & 1 & $3 / 2$ \\
${ }^{3} P_{2}$ & 1 & 1 & 2 & 3 & $5 / 2$ \\
${ }^{1} D_{2}$ & 2 & 0 & 2 & 2 & $3 / 2$ \\
${ }^{1} D_{2}$ & 2 & 0 & 2 & 2 & $5 / 2$ \\
\hline \hline
\end{tabular}

Table 10: Intermediate $3 n$ resonance positions together with the enhancement factor $\lambda_{3} P_{2}$ and $\lambda_{{ }_{1}}$ for a 6 channel calculation and $J^{\pi}=\frac{1}{2}^{+}$.

\begin{tabular}{||c|c|c||}
\hline \hline$\lambda P_{2}$ & $\lambda^{{ }}{ }_{D_{2}}$ & $E_{3 n}^{\text {res. }}[\mathrm{MeV}]$ \\
\hline \hline 3.43 & 6.43 & $0.64-i$ i 0.002 \\
3.00 & 6.00 & $12.90-i 4.46$ \\
2.00 & 5.00 & $16.91-i 22.63$ \\
1.00 & 4.00 & $11.94-i 34.46$ \\
1.00 & 3.00 & $3.97-i 41.41$ \\
1.00 & 2.00 & $-4.55-i 45.51$ \\
1.00 & 1.00 & $-14.29-i 48.34$ \\
\hline \hline
\end{tabular}




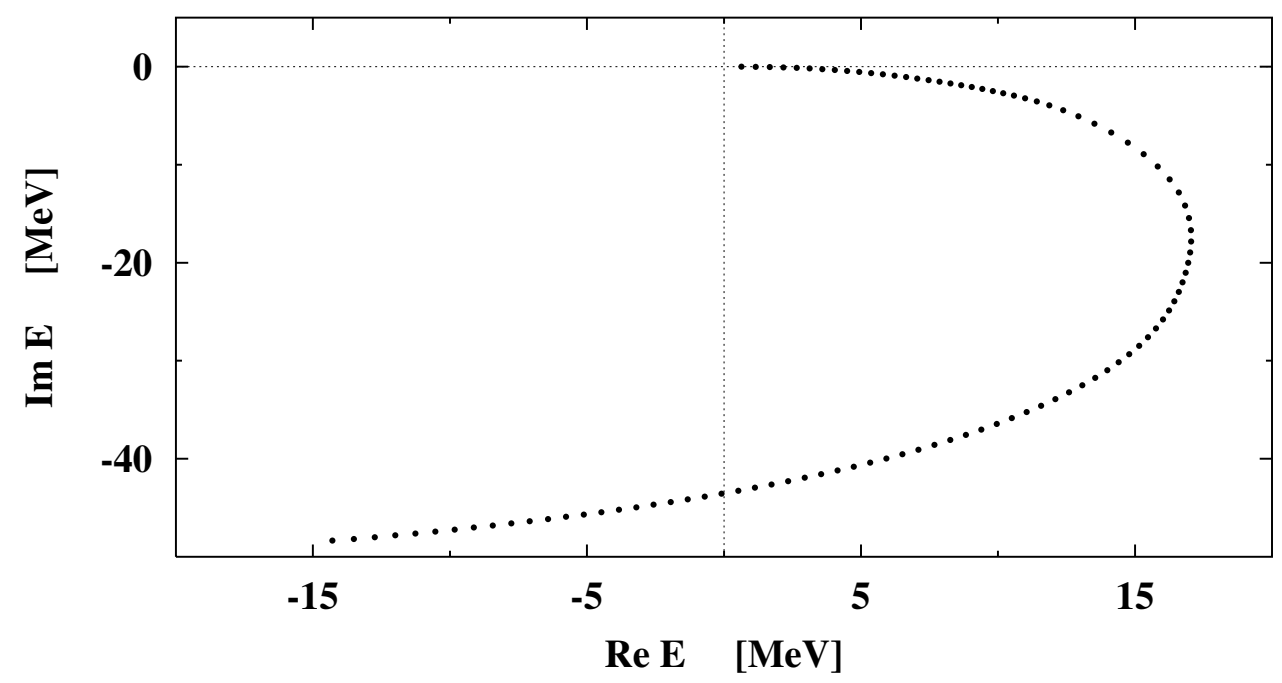

Figure 5: The resonance pole trajectory for the state $1 / 2^{+}$.

\section{Resonance Prediction via Padé}

The interesting idea to predict low lying resonances with the help of Padé approximants from a sequence of (auxiliary) bound state energies has been proposed in [22] and exemplified for instance in [23]. We also applied it to the system of $3 n$ 's. Assume, the $3 n$ binding energy is given in terms of a power series in an enhancement factor $\lambda$ as

$$
E(\lambda)=E\left(\lambda_{0}\right)+\left(\lambda-\lambda_{0}\right)^{\xi} a_{1}+\left(\lambda-\lambda_{0}\right)^{\xi+1} a_{2}+\cdots+\left(\lambda-\lambda_{0}\right)^{\xi+m-1} a_{m}
$$

where $\xi$ has still to be determined.In searching for resonances close to the threshold $E=0$ it is natural to choose $\lambda_{0}$ such that $E\left(\lambda_{0}\right)=0$. For two particles in this case it is known [22] that $\xi=1$ for a $S$-wave and $\xi=\frac{1}{2}$ for $P$-waves and higher ones. For a three-body system and the three-body break-up threshold we are not aware of an analytical insight into the value $\xi$. We determined $\xi$ numerically by approaching $E=0$ from both sides, $\lambda$ greater than $\lambda_{0}$ and $\lambda$ smaller than $\lambda_{0}$. We found $\xi=\frac{1}{2}$ within our numerical accuracy for all studied cases $J^{\pi}=1 / 2^{ \pm}$and $J^{\pi}=3 / 2^{ \pm}$. Choosing $m 3 n$ bound state energies close to $E=0$ one can determine $a_{1}, a_{2}, \cdots a_{m}$. To do that we solved the resulting $m$ equations by the method of singular value decomposition [24]. The power series (6) can then be rewritten in form of Padé approximants. We used a continued fraction expansion, which is equivalent to certain orders of approximants(see for instance [19]). Choosing for instance $m=6$ one can reach the Padé approximant of the order $[3,3]$. This turned out to be sufficient to predict the low lying resonances displayed in Tables 12,14,16 and 18. In addition we show the bound state energies in Tables 11,13,15 and 17 out of which the $a_{m}$ have been determined. There we also include the resonance positions determined directly from the analytically continued Faddeev equation. In the cases $J^{\pi}=\frac{3}{2}^{+}$and $\frac{1}{2}^{ \pm}$we changed two enhancement factors at the same time, but exactly by the same amount $\Delta \lambda$. In this case $\Delta \lambda$ is the expansion parameter in Eq. (6). The $a_{m}$ 's as well as the resonance energies are then determined either through the enhancement factors in one or the other state. The resulting resonance positions are exactly the same. The last rows in Tables 13, 15 and 17 show the two $\lambda_{0^{-}}$-values to many digits required to reach a significantly 
Table 11: The $J^{\pi}=\frac{3}{2}^{-}$bound state energies used as input for Padé [3,3]. The last row shows $\lambda_{0}$ and the resulting energy.

\begin{tabular}{||c|c|}
\hline \hline$\lambda_{{ } P_{2}}$ & $E_{B}^{3 n}[\mathrm{MeV}]$ \\
\hline \hline 3.41 & -4.79366 \\
3.36 & -3.28446 \\
3.31 & -1.89909 \\
3.26 & -0.65666 \\
3.25 & -0.42848 \\
3.24 & -0.20848 \\
3.2300873 & $-1.1 \times 10^{-8}$ \\
\hline \hline
\end{tabular}

Table 12: The $J^{\pi}=\frac{3}{2}^{-} 3 n$ resonance positions determined directly from the Faddeev equation in comparison to the Padé [3,3] prediction.

\begin{tabular}{||c|c|c||}
\hline \hline$\lambda{ }_{2}$ & $E_{\text {res }}^{3 n}[\mathrm{Me}]$ via Faddeev & Padé $[3,3][\mathrm{MeV}]$ \\
\hline \hline 3.22 & $0.20-i 0.003$ & $0.20-i 0.003$ \\
3.21 & $0.39-i 0.014$ & $0.39-i 0.012$ \\
3.20 & $0.57-i 0.03$ & $0.57-i 0.03$ \\
3.19 & $0.74-i 0.06$ & $0.74-i 0.05$ \\
3.18 & $0.91-i 0.09$ & $0.90-i 0.08$ \\
3.17 & $1.08-i 0.13$ & $1.06-i 0.12$ \\
3.16 & $1.24-i 0.18$ & $1.21-i 0.16$ \\
3.15 & $1.40-i 0.23$ & $1.35-i 0.22$ \\
3.14 & $1.56-i 0.28$ & $1.50-i 0.28$ \\
3.13 & $1.71-i 0.34$ & $1.63-i 0.35$ \\
\hline \hline
\end{tabular}

small $3 n$ binding energy. In all cases we see a very good agreement of the low lying resonances using the Padé method with the directly calculated ones. It is important to note that the $3 n$ bound state energies which serve as input have to be known to 5 digits in order to achieve the quoted Padé results. 
Table 13: The $J^{\pi}=\frac{3}{2}^{+}$bound state energies used as input for Padé [3,3]. For the last row see text.

\begin{tabular}{||c|c|c||}
\hline \hline$\lambda{ }_{P_{2}}$ & $\lambda{ } P_{0}$ & $E_{B}^{3 n}[\mathrm{MeV}]$ \\
\hline \hline 3.41 & 4.91 & -4.04286 \\
3.36 & 4.86 & -2.76896 \\
3.31 & 4.81 & -1.58830 \\
3.26 & 4.76 & -0.51213 \\
3.25 & 4.75 & -0.31107 \\
3.24 & 4.74 & -0.11532 \\
3.2339687436 & 4.7339687436 & $-1.4 \times 10^{-9}$ \\
\hline \hline
\end{tabular}

Table 14: The $J^{\pi}=\frac{3}{2}^{+} 3 n$ resonance positions determined directly from the Faddeev equation in comparison to the Padé $[3,3]$ prediction.

\begin{tabular}{||c|c|c|c||}
\hline \hline$\lambda_{{ } P_{2}}$ & $\lambda_{{ }^{3} P_{0}}$ & $E_{\text {res }}^{3 n}[\mathrm{MeV}]$ & Padé $[3,3][\mathrm{MeV}]$ \\
\hline \hline 3.22 & 4.72 & $0.26-i 0.0001$ & $0.26-i 0.0006$ \\
3.21 & 4.71 & $0.43-i 0.001$ & $0.43-i 0.003$ \\
3.20 & 4.70 & $0.60-i 0.004$ & $0.61-i 0.007$ \\
3.19 & 4.69 & $0.76-i 0.012$ & $0.77-i 0.013$ \\
3.18 & 4.68 & $0.92-i 0.024$ & $0.93-i 0.022$ \\
3.17 & 4.67 & $1.06-i 0.04$ & $1.08-i 0.03$ \\
3.16 & 4.66 & $1.21-i 0.06$ & $1.23-i 0.05$ \\
3.15 & 4.65 & $1.35-i 0.09$ & $1.37-i 0.07$ \\
3.14 & 4.64 & $1.49-i 0.13$ & $1.50-i 0.09$ \\
3.13 & 4.63 & $1.62-i 0.17$ & $1.63-i 0.11$ \\
3.12 & 4.62 & $1.76-i 0.21$ & $1.76-i 0.14$ \\
\hline \hline
\end{tabular}

Table 15: The $J^{\pi}=\frac{1}{2}^{-}$bound state energies used as input for Padé [3,3]. For the last row see text.

\begin{tabular}{||c|c|c||}
\hline \hline$\lambda^{3} P_{2}$ & $\lambda^{1} D_{2}$ & $E_{B}^{3 n}[\mathrm{MeV}]$ \\
\hline \hline 3.49 & 6.89 & -1.58303 \\
3.48 & 6.88 & -1.27353 \\
3.47 & 6.87 & -0.96991 \\
3.46 & 6.86 & -0.67262 \\
3.45 & 6.85 & -0.38224 \\
3.44 & 6.84 & -0.09983 \\
3.436380542 & 6.836380542 & $-1.8 \times 10^{-7}$ \\
\hline \hline
\end{tabular}


Table 16: The $J^{\pi}=\frac{1}{2}^{-} 3 n$ resonance positions determined directly from the Faddeev equation in comparison to the Padé $[3,3]$ prediction.

\begin{tabular}{||c|c|c|c||}
\hline \hline${ }^{{ } P_{2}}$ & $\lambda_{{ } D_{2}}$ & $E_{\text {res }}^{3 n}[\mathrm{MeV}]$ via Faddeev & Padé $[3,3][\mathrm{MeV}]$ \\
\hline \hline 3.43 & 6.83 & $0.17-i 0.001$ & $0.17-i 0.001$ \\
3.42 & 6.82 & $0.43-i 0.009$ & $0.43-i 0.01$ \\
3.41 & 6.81 & $0.69-i 0.02$ & $0.69-i 0.03$ \\
3.40 & 6.80 & $0.94-i 0.05$ & $0.94-i 0.06$ \\
3.39 & 6.79 & $1.19-i 0.07$ & $1.19-i 0.09$ \\
3.38 & 6.78 & $1.43-i 0.10$ & $1.44-i 0.13$ \\
3.37 & 6.77 & $1.67-i 0.14$ & $1.69-i 0.18$ \\
3.36 & 6.76 & $1.91-i 0.18$ & $1.94-i 0.23$ \\
\hline \hline
\end{tabular}

Table 17: The $J^{\pi}=\frac{1}{2}^{+}$bound state energies used as input for Padé [3,3]. For the last row see text.

\begin{tabular}{||c|c|c||}
\hline \hline$\lambda^{3} P_{2}$ & $\lambda{ }_{D_{2}}$ & $E_{B}^{3 n}[\mathrm{MeV}]$ \\
\hline \hline 3.50 & 6.90 & -7.37894 \\
3.45 & 6.85 & -5.02695 \\
3.41 & 6.81 & -3.22567 \\
3.36 & 6.76 & -1.08684 \\
3.35 & 6.75 & -0.67596 \\
3.34 & 6.74 & -0.27141 \\
3.333194193 & 6.733194193 & $-2.0 \times 10^{-7}$ \\
\hline \hline
\end{tabular}


Table 18: The $J^{\pi}=\frac{1}{2}^{+} 3 n$ resonance positions determined directly from Faddeev equation in comparison to Padé [3,3] prediction.

\begin{tabular}{||c|c|c|c||}
\hline \hline$\lambda{ }_{P_{2}}$ & $\lambda_{{ } D_{2}}$ & $E_{\text {res }}^{3 n}[\mathrm{MeV}]$ via Faddeev & Padé $[3,3][\mathrm{MeV}]$ \\
\hline \hline 3.33 & 6.73 & $0.13-i 0.0000$ & $0.13-i 0.0002$ \\
3.32 & 6.72 & $0.52-i 0.0007$ & $0.52-i 0.003$ \\
3.31 & 6.71 & $0.90-i 0.004$ & $0.90-i 0.007$ \\
3.29 & 6.70 & $1.27-i 0.009$ & $1.28-i 0.011$ \\
3.28 & 6.69 & $1.64-i 0.02$ & $1.66-i 0.01$ \\
3.27 & 6.68 & $2.00-i 0.03$ & $2.02-i 0.02$ \\
3.26 & 6.67 & $2.35-i 0.05$ & $2.38-i 0.02$ \\
3.25 & 6.66 & $2.70-i 0.07$ & $2.73-i 0.03$ \\
3.35 & 6.65 & $3.04-i 0.09$ & $3.07-i 0.03$ \\
\hline \hline
\end{tabular}

\section{Summary}

We analytically continued the Faddeev equation for three neutrons into the unphysical sheet of the complex energy plane adjacent to the positive real energy axis. The nn forces are of finite rank and were kept in the partial waves ${ }^{1} S_{0},{ }^{3} P_{0},{ }^{3} P_{1},{ }^{3} P_{2},{ }^{1} D_{2}$. This leads up to 13 channel calculations. The ${ }^{3} P_{1} n n$ force is repulsive and its effect turned out to be rather small in relation to the combined effects of all the other forces. The analytical continuation is performed via a contour deformation of the path of integration. The strategy is to artificially enhance the nn forces such that a $3 \mathrm{n}$ bound state is generated. Then by reducing that enhancement one follows the energy eigenvalue of the homogeneous 3n Faddeev equation which traces out a trajectory. After having reached the $3 \mathrm{n}$ break-up threshold $E=0$ that trajectory dives into the unphysical energy sheet. The final positions for the physical strength turned out for the four cases studied, $J^{\pi}=1 / 2^{ \pm}$and $J^{\pi}=3 / 2^{ \pm}$, to be far away from the positive real energy axis. Though the nn forces employed are not of the quality of the modern high precision $N N$ potentials it appears unlikely that those modern forces would lead to totally different results. Thus we conjecture that $3 \mathrm{n}$ resonances close to the physical region will not exist. This conjecture is also supported by the findings in [16].

These results gained through the analytically continued Faddeev equation have been tested numerically using Padé approximants. In this method one generates a set of auxiliary $3 \mathrm{n}$ bound state energies close to $E=0$ for different enhancement factors $\lambda$. This determines the first few coefficients in a power series expansion of $E(\lambda)$. This again leads to the coefficients of Padé approximants which can be used to predict low lying $3 \mathrm{n}$ resonances. The resulting values agreed very well with the ones determined via the Faddeev equation. 
Table 19: The parameters of our $n n$ potential. The units of these parameters are $\mathrm{fm}^{-1}$ for $\beta_{i j}$, $\mathrm{MeV} \mathrm{fm}{ }^{-2 l-1}$ for $\lambda_{i}$ and the $\gamma_{i}$ are dimensionless.

\begin{tabular}{||c|c|c|c|c|c||}
\hline \hline & ${ }^{1} S_{0}$ & ${ }^{3} P_{0}$ & ${ }^{3} P_{1}$ & ${ }^{3} P_{2}$ & ${ }^{1} D_{2}$ \\
\hline \hline$\beta_{11}$ & 0.8131678 & 0.8322894 & 0.9713441 & 1.977127 & 2.522169 \\
$\beta_{12}$ & 1.288463 & 1.262785 & 2.180297 & 3.147108 & 1.651999 \\
$\beta_{21}$ & 7.496476 & 2.645783 & & & \\
$\beta_{22}$ & 1.661389 & & & & \\
$\gamma_{1}$ & 2.698168 & 6.397468 & 31.97981 & 5.242138 & -0.2668325 \\
$\gamma_{2}$ & 0.3270664 & & & & \\
$\lambda_{1}$ & -17.87098 & -12.10082 & 59.89249 & -1477.246 & -918408.7 \\
$\lambda_{2}$ & 82710.93 & 277071.8 & & & \\
\hline \hline
\end{tabular}

Table 20: The $n n$ effective range parameter $r$ and the scattering length $a$ in units of fm for the ${ }^{1} S_{0}$-state in comparison with experimental results.

\begin{tabular}{||c|c|c||}
\hline \hline & $r$ & $a$ \\
\hline \hline our potential & 2.85 & -19.08 \\
exp. data [21] & $2.83 \pm 0.11$ & $-18.6 \pm 0.5$ \\
\hline \hline
\end{tabular}




\section{Acknowledgements}

We would like to thank Y. Koike for very helpful hints at the beginning of the study. A. Hemmdan would like to thank the Institut für Theoretische Physik II of the Ruhr-Universität Bochum for the very kind hospitality. This research was supported by the Egyptian government under a fellowship in the Channel System.

\section{Appendix A}

The finite rank potential we use has the following form [25]

$$
V(p, p \prime)=\sum_{i=1}^{2} g_{i}(p) \lambda_{i} g_{i}(p \prime)
$$

where

$$
\begin{aligned}
& g_{1}(p)=\frac{p^{l}}{\left(p^{2}+\beta_{11}^{2}\right)^{l+1}}+\frac{p^{l+2}}{\left(p^{2}+\beta_{12}^{2}\right)^{l+2}} \gamma_{1} \\
& g_{2}(p)=\frac{p^{l+2}}{\left(p^{2}+\beta_{21}^{2}\right)^{l+2}}+\frac{p^{l+4}}{\left(p^{2}+\beta_{22}^{2}\right)^{l+3}} \gamma_{2}
\end{aligned}
$$

The $l$-dependent parameters of the potential form factors are displayed in Table 19 . The resulting phase shifts of the $\mathrm{nn}$ system for the partial waves ${ }^{1} S_{0},{ }^{3} P_{0},{ }^{3} P_{1},{ }^{3} P_{2}$ and ${ }^{1} D_{2}$ are compared to $p p$ phase shift values (without Coulomb) given in SAID [21] in Fig. 6. In Table 20 we show the scattering length $a$ and the effective range $r$ for the $n n$ system in the state ${ }^{1} S_{0}$. 

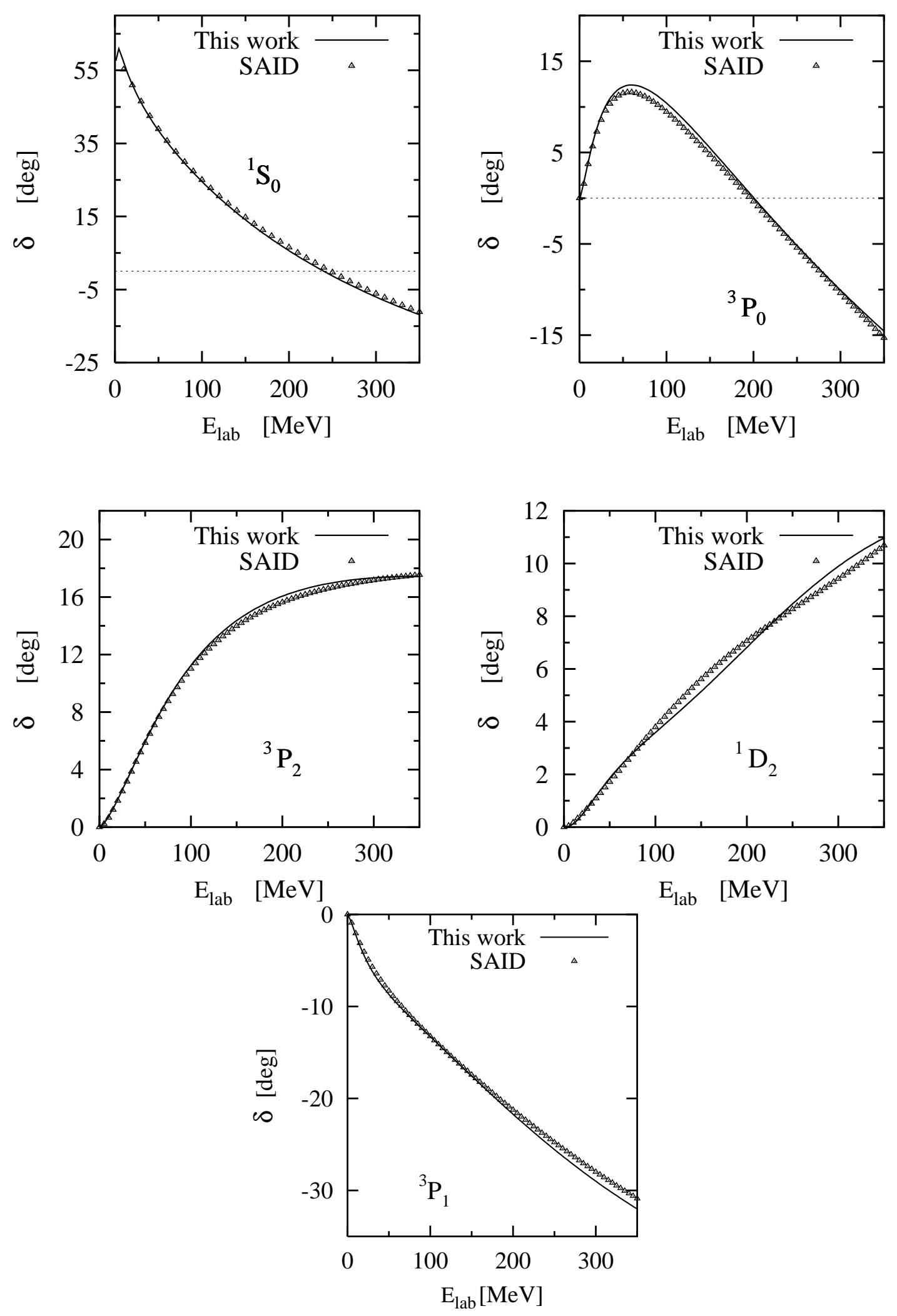

Figure 6: The calculated $n n$ phase shifts compared to SAID [21] $p p$ phase shift values (without Coulomb). 


\section{References}

1. D. R. Tilley, H. R. Weller and H. H. Hasan, Nucl. Phys. A474, 1 (1987).

2. M. Yuly et al., Phys. Rev. C55, 1848 (1997).

3. J. Sperinde, D. Frederickson, R. Hinkins, V. Prez-Mendez and B. Smith, Phys. Lett. 32B, 185 (1970).

4. A. Setz et al., Nucl. Phys. A457, 669 (1986).

5. F. M. Marques et al., Phys. Rev. C65, 044006 (2002).

6. S. A. Coon and H. K. Han, Few-Body System 30, 131 (2001).

7. B. S. Pudliner et al., Phys. Rev. C56, 1720 (1997).

8. W. Glöckle. Phys. Rev. C18, 564 (1978).

9. K. Möller and Yu. Orlov, Sov. J. Part. Nucl. 20, 569 (1989).

10. R. Offermann and W. Glöckle, Nucl. Phys. A318, 138 (1979).

11. J. R. Reid, Ann. Phys. 50, 411 (1968).

12. S. A. Sofianos, S. A. Rakityansky and G. P. Vermaark, J. Phys. G: Nucl. Part. Phys. 23, 1619 (1997).

13. A. Csoto, H. Oberhummer and R. Pichler, Phys. Rev. C53, 1589 (1986).

14. D. R. Thompson, M. LeMere and Y. C. Tang, Nucl. Phys. A268, 53 (1977); I. Reichstein and Y.C.Tang, Nucl. Phys. A158, 529 (1970); A. Csoto, Phys. Rev. C48, 165 (1993).

15. P. Heiss and H. H. Hackenbroich, Phys. Lett. 30B, 373 (1969); A. Csoto, R. G. Lovas and A. T. Kruppa, Phys. Lett. 70, 1389 (1993).

16. H. Witała and W. Glöckle, Phys. Rev. C60, 024002 (1999).

17. D. Gogny, P. Pires and R. de Tourreil, Phys. Lett. 32B, 59, (1970).

18. V. G. Stoks et al., Phys. Rev. C49, 2950 (1994).

19. W. Glöckle, The Quantum Mechanical Few-Body Problem, Springer Verlag, Heidelberg, 1983.

20. A. Matsuyama and K. Yazaki, Nucl. Phys. A534, 620 (1991).

21. http://NN-online.sci.kun.nl/index.htm.

22. V. I. Kukulin et al., Sov. J. Nucl. Phys. 92, 421 (1979).

23. N. Tanaka, Y. Suzuki, K. Varga and R. G. Lovas, Phys. Rev. C59, 1391 (1999).

24. Numerical Recipes, Cambridge University Press (1992).

25. W. Schweiger, W. Plessas, L. P. Kok, H. van Haeringen, Phys. Rev. C 27, 515 (1983). 